\title{
On the Physics of Type I X-Ray Bursts on Accreting Neutron Stars at High Accretion Rates
}

\section{Citation}

Cooper, Randall L., and Ramesh Narayan. 2006. "On the Physics of Type I X-Ray Bursts on Accreting Neutron Stars at High Accretion Rates." The Astrophysical Journal 648 (2): L123-26. https://doi.org/10.1086/508167.

\section{Permanent link}

http://nrs.harvard.edu/urn-3:HUL.InstRepos:41384974

\section{Terms of Use}

This article was downloaded from Harvard University's DASH repository, and is made available under the terms and conditions applicable to Other Posted Material, as set forth at http:// nrs.harvard.edu/urn-3:HUL.InstRepos:dash.current.terms-of-use\#LAA

\section{Share Your Story}

The Harvard community has made this article openly available.

Please share how this access benefits you. Submit a story.

Accessibility 
DRAFT VERSION SEPTEMBER 19, 2018

Preprint typeset using $\mathrm{LT}_{\mathrm{E}} \mathrm{X}$ style emulateapj v. 6/22/04

\title{
ON THE PHYSICS OF TYPE I X-RAY BURSTS ON ACCRETING NEUTRON STARS AT HIGH ACCRETION RATES
}

\author{
RANDALL L. COOPER AND RAMESH NARAYAN \\ Harvard-Smithsonian Center for Astrophysics, 60 Garden Street, Cambridge, MA 02138 \\ Draft version September 19, 2018
}

\begin{abstract}
We investigate the effect of the hot CNO cycle breakout reaction ${ }^{15} \mathrm{O}(\alpha, \gamma){ }^{19} \mathrm{Ne}$ on the occurrence of type I $\mathrm{X}$-ray bursts on accreting neutron stars. For $f_{\mathrm{rp}} \lesssim 0.1$, where $f_{\mathrm{rp}}$ is a dimensionless factor by which we multiply the ${ }^{15} \mathrm{O}(\alpha, \gamma){ }^{19} \mathrm{Ne}$ reaction rate of Caughlan \& Fowler (1988), our model predicts that bursts should occur only for accretion rates $\dot{M}$ below a critical value $\approx 0.3 \dot{M}_{\mathrm{Edd}}$. This agrees with observations. For larger values of $f_{\text {rp }}$, including the standard choice $f_{\text {rp }}=1$, the model switches to a new regime in which bursts occur all the way up to $\dot{M} \approx \dot{M}_{\mathrm{Edd}}$. Since the latter regime disagrees with observations, we suggest that the true ${ }^{15} \mathrm{O}(\alpha, \gamma){ }^{19} \mathrm{Ne}$ reaction rate is lower than usually assumed.

Subject headings: dense matter — nuclear reactions, nucleosynthesis, abundances — stars: neutron — X-rays: binaries - X-rays: bursts
\end{abstract}

\section{INTRODUCTION}

Type I X-ray bursts are thermonuclear explosions that occur on accreting neutron stars in low-mass X-ray binaries. They are triggered by thermally unstable $\mathrm{H}$ or $\mathrm{He}$ burning near the stellar surface (for reviews, see Cumming 2004; Strohmaver \& Bildsten 2006). For systems with accretion rates $\dot{M} \lesssim 0.1 \dot{M}_{\text {Edd }}$, where $\dot{M}_{\text {Edd }}$ denotes the mass accretion rate at which the accretion luminosity is equal to the Eddington limit, the basic physics of the burst onset is well understood to be that of the thin shell thermal instability (Schwarzschild \& Härm 1965; Hansen \& van Horn 1975), and theoretical models have been rather successful at reproducing the gross characteristics of burst observations in this regime (e.g., Fuiimoto et al. 1981; Fushiki \& Lamb 1987; Cumming \& Bildsten 2000; Naravan \& Hevl 2003).

This Letter addresses a longstanding problem that afflicts nearly all burst models when the accretion rate $\dot{M} \geq 0.1 \dot{M}_{\text {Edd }}$. Both simple one-zone burst models (Fuiimoto et al. 1981; Paczyński 1983; Bildsten 1998; Heger et al. 2005) and sophisticated time-dependent multi-zone models (Avasli \& Joss 1982; Taam et al. 1996; Fisker et al. 2003; Heger et al. 2005) predict that bursts should occur for all $\dot{M}$ up to $\approx \dot{M}_{\text {Edd }}$. Observations, however, indicate that bursts do not occur for $\dot{M} \geq 0.3 \dot{M}_{\text {Edd }}$ (van Paradiis et al. 1979, 1988; Cornelisse et al. 2003; Remillard et al. 2006). Furthermore, van Paradijs et al. (1988) found that, for $0.1 \lesssim \dot{M} / \dot{M}_{\text {Edd }} \lesssim 0.3$, a significant fraction of the accreted plasma burns stably between consecutive bursts, leading to large values $\gtrsim 1000$ of the parameter $\alpha$, the accretion energy released between successive bursts divided by the nuclear energy released during a burst. Most theoretical models, on the other hand, predict that nearly all of the accreted matter burns unstably during bursts, giving a nearly constant $\alpha<100$ at all $\dot{M}$.

Naravan \& Hevl (2003, hereafter NH03) developed a global linear stability analysis of the accreted plasma on the surface of a neutron star. They discovered a new regime of unstable nuclear burning for $0.1 \lesssim \dot{M} / \dot{M}_{\text {Edd }} \lesssim 0.3$ that they referred to as "delayed mixed bursts." Their model reproduced both the occurrence of considerable stable burning preceding a burst (leading to values of $\alpha \gtrsim 1000$ ) and the absence

Electronic address: rcooper@cfa.harvard.edu,rnarayan@cfa.harvard.edu of bursts for $\dot{M} \gtrsim 0.3 \dot{M}_{\text {Edd }}$, in agreement with observations. However, the complexity of their model made it difficult to understand the basic physics behind delayed mixed bursts and to identify the reasons why their results differed from those of other theoretical models at high accretion rates. To remedy this, Cooper \& Naravan (2006, hereafter CN06) constructed a simple two-zone model that helped elucidate the physics of delayed mixed bursts. They showed that the competition between nuclear heating due to triple- $\alpha$ reactions and hot $\mathrm{CNO}$ cycle $\mathrm{H}$ burning on the one hand, and radiative cooling via photon diffusion and emission on the other hand, drives an overstability that eventually triggers a thin-shell thermal instability and hence a delayed mixed burst. They asserted that $\mathrm{H}$ burning via the temperature-independent hot $\mathrm{CNO}$ cycle, augmented by the extra seed nuclei produced from stable $\mathrm{He}$ burning, significantly lowers the temperature sensitivity of the total nuclear energy generation rate to such an extent as to suppress the thin-shell thermal instability for $\dot{M} \gtrsim 0.3 \dot{M}_{\text {Edd }}$. Therefore, $\mathrm{H}$ burning via the hot $\mathrm{CNO}$ cycle is ultimately responsible for the lower critical $\dot{M}$ above which bursts do not occur in nature.

The above argument perhaps explains why one-zone models fail, since the models generally focus only on He burning and make large approximations with respect to $\mathrm{H}$ burning. But why do detailed time-dependent multi-zone models with large reaction networks also perform poorly in relation to observations? These models are much more sophisticated than the models of NH03 and CN06 and consequently ought to perform better, whereas in fact the latter models agree much better with observations. CN06 hypothesized that the time-dependent multi-zone burst models may have used too large a rate for the experimentally poorlyconstrained hot CNO cycle breakout reaction ${ }^{15} \mathrm{O}(\alpha, \gamma){ }^{19} \mathrm{Ne}$ (Wagoner 1969; Wallace \& Wooslev 1981; Langanke et al. 1986; Wiescher et al. 1999; Fisker et al. 2006). We test this hypothesis. We begin in $\$ 2$ with a description of the model, and we present the results of the model in $\$ 3$ We discuss the results in $\$ 4$ and we conclude in $\$ 5$

\section{THE MODEL}

We use the general-relativistic global linear stability analysis of Cooper \& Naravan (2005), which is an expanded and improved version of the model of $\mathrm{NH} 03$, to determine the sta- 
bility of nuclear burning on accreting neutron stars. We assume that matter accretes spherically onto a neutron star of gravitational mass $M=1.4 M_{\odot}$ and areal radius $R=10.4 \mathrm{~km}$ at a rate $\dot{M}$, where $\dot{M}$ is the rest mass accreted per unit time as measured by an observer at infinity. We set the composition of the accreted matter to be that of the Sun, such that at the neutron star surface the $\mathrm{H}$ mass fraction $X_{\text {out }}=0.7, \mathrm{He}$ mass fraction $Y_{\text {out }}=0.28, \mathrm{CNO}$ mass fraction $Z_{\mathrm{CNO} \text {, out }}=0.016$, and heavy element fraction $Z_{\text {out }}=0.004$, where $Z$ refers to all metals other than CNO. In this section, we describe the modifications to the theoretical model.

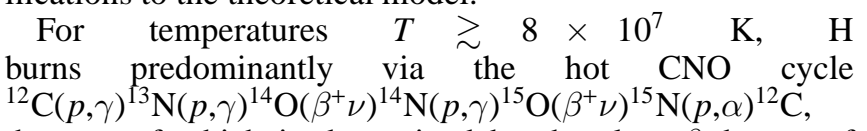
the rate of which is determined by the slow $\beta$-decays of ${ }^{14} \mathrm{O}\left(t_{1 / 2}=70.6 \mathrm{~s}\right)$ and ${ }^{15} \mathrm{O}\left(t_{1 / 2}=122 \mathrm{~s}\right.$ ) (Hovle \& Fowler 1965). During the hot CNO cycle, essentially all of the $\mathrm{CNO}$ ions are converted to ${ }^{14} \mathrm{O}$ and ${ }^{15} \mathrm{O}$. For the range of accretion rates we consider in this investigation, the primary breakout reaction from the hot $\mathrm{CNO}$ cycle into the rp-process of Wallace \& Wooslev (1981) is ${ }^{15} \mathrm{O}(\alpha, \gamma){ }^{19} \mathrm{Ne}$ (Görres et al. 1995; Hahn et al. 1996; Schatz et al. 1999). If this breakout reaction occurs, the ${ }^{19} \mathrm{Ne}\left(t_{1 / 2}=17.2 \mathrm{~s}\right)$ produced in this reaction can $\beta$-decay and return to the hot $\mathrm{CNO}$ cycle via ${ }^{19} \mathrm{Ne}\left(\beta^{+} \nu\right){ }^{19} \mathrm{~F}(p, \alpha){ }^{16} \mathrm{O}(p, \gamma){ }^{17} \mathrm{~F}(p, \gamma){ }^{18} \mathrm{Ne}\left(\beta^{+} \nu\right){ }^{18} \mathrm{~F}(p$, $\alpha)^{15} \mathrm{O}$, the effect of which is only to expedite the hot CNO cycle by a factor $\lesssim 1.6$. We ignore this effect in our model. If ${ }^{19} \mathrm{Ne}$ captures a proton, however, the resulting ${ }^{20} \mathrm{Na}$ ion can never return to the hot CNO cycle (Wallace \& Woosley 1981). The fate of a ${ }^{19} \mathrm{Ne}$ ion thus depends on whether or not it captures a proton before it can $\beta$-decay. Therefore, the probability that a ${ }^{19} \mathrm{Ne}$ ion is removed from the hot $\mathrm{CNO}$ cycle is

$$
R=\frac{\rho X \lambda_{p \gamma}\left({ }^{19} \mathrm{Ne}\right)}{\rho X \lambda_{p \gamma}\left({ }^{19} \mathrm{Ne}\right)+\lambda_{\beta^{+}}\left({ }^{19} \mathrm{Ne}\right)},
$$

where $\rho$ is the density, $X$ is the $\mathrm{H}$ mass fraction, $\lambda_{p \gamma}\left({ }^{19} \mathrm{Ne}\right)$ is the ${ }^{19} \mathrm{Ne}(p, \gamma){ }^{20} \mathrm{Na}$ reaction rate from Caughlan \& Fowler (1988, hereafter CF88) with electron screening from Dewitt et al. (1973), and $\lambda_{\beta^{+}}\left({ }^{19} \mathrm{Ne}\right)=\ln 2 / t_{1 / 2}$ is the ${ }^{19} \mathrm{Ne} \beta$ decay rate. If ${ }^{19} \mathrm{Ne}$ captures a proton, further proton captures will ensue until the nuclear flow reaches the first waiting point of the rp-process, which we assume to be ${ }^{24} \mathrm{Si}$ (Wiescher et al. 1998). The ${ }^{15} \mathrm{O}(\alpha, \gamma){ }^{19} \mathrm{Ne}$ reaction is the slowest reaction of this sequence, and so its rate governs the total reaction rate of this flow. Therefore, we follow Wiescher et al. (1999) and approximate the total nuclear energy generation rate of the hot $\mathrm{CNO}$ cycle breakout reaction sequence by

$$
\epsilon_{\mathrm{rp}}=24 E_{\mathrm{rp}}^{*}\left(\frac{Y}{4}\right)\left(\frac{Z_{\mathrm{CNO}}}{15}\right) \rho R \lambda_{\alpha \gamma}\left({ }^{15} \mathrm{O}\right) \times f_{\mathrm{rp}},
$$

where $E_{\mathrm{rp}}^{*}=Q_{\mathrm{rp}} / 24 m_{\mathrm{p}}=1.2 \times 10^{18} \mathrm{ergs} \mathrm{g}^{-1}, Y$ is the He mass fraction, $Z_{\mathrm{CNO}}$ is the mass fraction of $\mathrm{CNO}$ elements, and $\lambda_{\alpha \gamma}\left({ }^{15} \mathrm{O}\right)$ is the ${ }^{15} \mathrm{O}(\alpha, \gamma){ }^{19} \mathrm{Ne}$ reaction rate from $\mathrm{CF} 88$, which is based on the rate derived by Langanke et al. (1986). For the temperatures $T \lesssim 6 \times 10^{8} \mathrm{~K}$ we consider in this work, the resonant contribution of the $4.03 \mathrm{MeV}$ state in ${ }^{19} \mathrm{Ne}$ dominates the ${ }^{15} \mathrm{O}(\alpha, \gamma){ }^{19} \mathrm{Ne}$ reaction rate (Langanke et al. 1986). The reaction rate of this contribution is directly proportional to the $\alpha$ width $\Gamma_{\alpha}$. However, $\Gamma_{\alpha}$ is very difficult to measure experimentally, and so the true $\lambda_{\alpha \gamma}\left({ }^{15} \mathrm{O}\right)$ is highly uncertain (for recent progress on experimental constraints, see, e.g., Tan et al. 2005; Kanungo et al. 2006). To account for this uncertainty, we multiply the CF88 rate by a dimensionless free parameter $f_{\text {rp }}$ such that $f_{\text {rp }}=1$ corresponds the the usual rate of CF88. We omit the electron screening contribution to the reaction rate since the enhancement factor itself depends on the relatively unconstrained resonance strength (Salpeter \& van Horn 1969; Mitler 1977). Instead, we simply absorb this contribution into $f_{\mathrm{rd}}$. Equations (6), (8), (9), and (10) of Cooper \& Naravan (2005) thus become

$$
\begin{gathered}
e^{-2 \Phi / c^{2}} \frac{\partial}{\partial \Sigma}\left(\frac{F r^{2}}{R^{2}} e^{2 \Phi / c^{2}}\right)=T \frac{d s}{d t}-\left(\epsilon_{\mathrm{H}}+\epsilon_{\mathrm{He}}+\epsilon_{\mathrm{rp}}+\epsilon_{\mathrm{N}}-\epsilon_{\nu}\right) \\
\frac{d X}{d t}=-\frac{\epsilon_{\mathrm{H}}}{E_{\mathrm{H}}^{*}}-\frac{5}{24} \frac{\epsilon_{\mathrm{rp}}}{E_{\mathrm{rp}}^{*}} \\
\frac{d Y}{d t}=\frac{\epsilon_{\mathrm{H}}}{E_{\mathrm{H}}^{*}}-\frac{\epsilon_{\mathrm{He}}}{E_{\mathrm{He}}^{*}}-\frac{4}{24} \frac{\epsilon_{\mathrm{rp}}}{E_{\mathrm{rp}}^{*}} \\
\frac{d Z_{\mathrm{CNO}}}{d t}=\frac{\epsilon_{\mathrm{He}}}{E_{\mathrm{He}}^{*}}-\frac{15}{24} \frac{\epsilon_{\mathrm{rp}}}{E_{\mathrm{rp}}^{*}}
\end{gathered}
$$

See Cooper \& Narayan (2005) for the definitions of the various symbols. Note that the factor of 5 in equation (4) accounts for the five proton captures needed to reach ${ }^{24} \mathrm{Si}$. For a substantial portion of the accretion rates we consider in this work, the nuclear flow due to stable burning extends well beyond the relatively small number of isotopes included in our limited network (e.g., Schatz et al. 1999; Fisker et al. 2005). However, the contributions from $\mathrm{H}$ and He burning dominate the total nuclear energy generation rate and set the thermal profile of the accreted layer, and we calculate these contributions to high accuracy. Consequently, the thermal profiles resulting from our network compare very well with those of Schatz et al. (1999) for the range of accretion rates in which our calculations overlap. Furthermore, we are interested only in the physics of the burst onset, for which only hot CNO cycle, triple- $\alpha$, and breakout reactions are important. Thus, our limited network should be adequate for our purposes.

\section{RESULTS}

Figure 1 shows the values of the dimensionless quantity $\alpha$ as a function of the Eddington-scaled accretion rate $l_{\text {acc }} \equiv$ $\dot{M} / \dot{M}_{\text {Edd }}$ for five different values of $f_{\text {rp }}$, where $\alpha$ is defined as the accretion energy released between successive bursts divided by the nuclear energy released during a burst. We follow NH03 and call a type I X-ray burst "prompt" if $\alpha \lesssim 100$ and "delayed" if $\alpha \gg 100$. The case $f_{\mathrm{rp}}=0$ omits the breakout reaction sequence entirely, and so this calculation is representative of the results of $\mathrm{NH03}$ and $\mathrm{CN} 06$. The models gives prompt bursts for $l_{\text {acc }} \lesssim 0.15$ and delayed bursts over the range $l_{\text {acc }} \approx 0.15-0.3$. For low values of $f_{\text {rp }} \lesssim 0.1$, the effects of breakout reactions from the hot CNO cycle on the occurrence of type I X-ray bursts are minor, and the models of NH03 and CN06 continue to provide an adequate description of the nuclear physics that precedes bursts. However, the situation changes for $f_{\mathrm{rp}} \gtrsim 0.1$. The critical $l_{\text {acc }}$ above which delayed mixed bursts cease now significantly decreases, and the range of accretion rates in which delayed mixed bursts occur becomes notably truncated as well. More importantly, a new burst regime appears at accretion rates close to $\dot{M}_{\text {Edd }}$, where the bursts have $\alpha<100$ and are hence prompt. This regime of bursts near the Eddington limit was hypothesized by CN06, but it was not present in either the NH03 or CN06 models due to the exclusion of hot CNO cycle breakout reactions. The usual delayed mixed burst regime and this new 


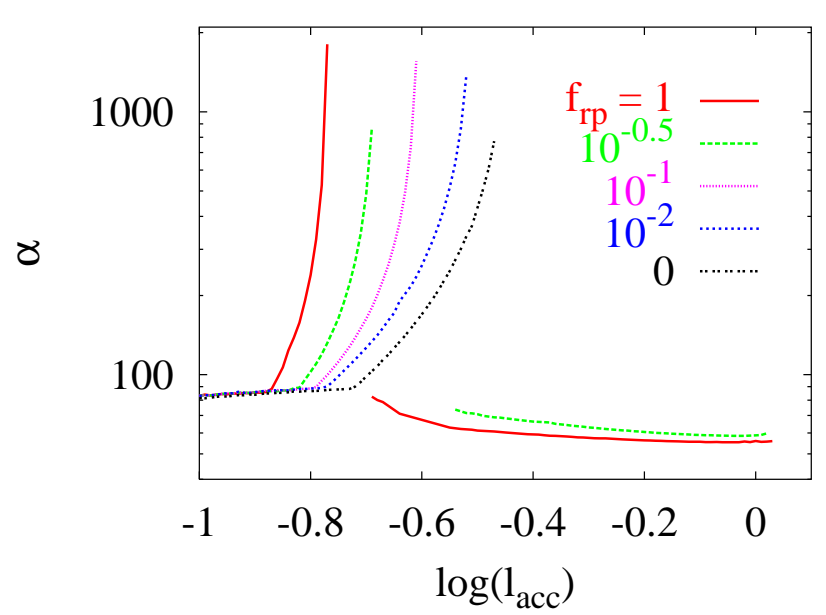

FIG. 1.- Plot shows $\alpha$-values of type I X-ray bursts as a function of the Eddington-scaled accretion rate $l_{\text {acc }} \equiv \dot{M} / \dot{M}_{\text {Edd }}$ for five different values of $f_{\mathrm{rp}}$, the dimensionless factor by which we multiply the ${ }^{15} \mathrm{O}(\alpha, \gamma){ }^{19} \mathrm{Ne}$ reaction rate from CF88.

prompt mixed burst regime are separated by a short range of accretion rates in which bursts do not occur. These results obtained with larger values of $f_{\text {ro }}$ agree rather well with previous theoretical models (Fuiimoto et al. 1981; Avasli \& Joss 1982; Paczvński 1983; Taam 1985; Taam et al. 1996; Bildsten 1998; Fisker et al. 2003; Heger et al. 2005), most of which predicted bursts to occur up to roughly the Eddington limit. Figure 1 thus explains the origin of the differences between those models and the models of NH03 and CN06. If the breakout reaction rate is large, say $f_{\mathrm{rp}} \gtrsim 0.1$, the results agree with most published models (which include breakout reactions in full strength), and if the rate is small, $f_{\text {rp }} \lesssim 0.1$, the results are similar to those obtained by $\mathrm{NH} 03$ and $\mathrm{CN} 06$ (who effectively set $f_{\mathrm{rp}}=0$ ). We reiterate that the latter results agree much better with observations.

\section{DISCUSSION}

For $l_{\text {acc }} \lesssim 0.1$, the temperatures reached during steady-state nuclear burning in the accreted layer are too low for significant leakage out of the hot CNO cycle via breakout reactions, and so these reactions have a negligible effect on the onset of type I X-ray bursts, regardless of the true ${ }^{15} \mathrm{O}(\alpha, \gamma){ }^{19} \mathrm{Ne}$ cross section. However, breakout reactions should affect the nuclear flow during the burst itself, and hence they could influence the burst lightcurve and possibly also the onset of subsequent bursts (Fisker et al. 2006). CN06 showed that the delayed mixed bursts of $\mathrm{NH} 03$ occur when $\Sigma_{\mathrm{H}}$, the column depth at which $\mathrm{H}$ is depleted via stable nuclear burning as measured from the stellar surface, is less than but close to $\Sigma_{\mathrm{He}}$, the column depth at which He is depleted via stable nuclear burning. The hot CNO cycle breakout reaction sequence (i) eliminates seed nuclei from the hot $\mathrm{CNO}$ cycle which slows $\mathrm{H}$ burning, thereby increasing $\Sigma_{\mathrm{H}}$, and (ii) provides an additional pathway by which He may burn, thereby decreasing $\Sigma_{\mathrm{He}}$. Consequently, one expects the regime of delayed mixed bursts to occur at lower $l_{\text {acc }}$ if breakout reactions are included. Furthermore, CN06 showed that the interplay between $\mathrm{H}$ burning via the hot $\mathrm{CNO}$ cycle and He burning via triple- $\alpha$ reactions is integral to generating the oscillations that precede delayed mixed bursts. Breakout reactions diminish this interplay by eliminating the hot $\mathrm{CNO}$ cycle seed nuclei and should there- fore reduce the range of accretion rates over which delayed mixed bursts occur. Figure 1 illustrates both of these effects (compare $f_{\text {rp }}=1$ with $f_{\text {rp }}=0$ ).

For accretion rates above those at which delayed mixed bursts occur, NH03 found that nuclear burning is always stable and therefore bursts do not occur, in accord with observations van Paradijs et al. 1979, 1988; Cornelisse et al. 2003; Remillard et al. 2006). According to their model, for $0.3 \lesssim$ $l_{\text {acc }} \lesssim 1$, steady-state $\mathrm{H}$ burning via the hot $\mathrm{CNO}$ cycle increases the effective radiative cooling rate and thereby suppresses a He-triggered thin-shell thermal instability (CN06). However, their model did not include breakout reactions. If the ${ }^{15} \mathrm{O}(\alpha, \gamma){ }^{19} \mathrm{Ne}$ breakout reaction rate is significant in this regime, this reaction will suppress hot $\mathrm{CNO}$ cycle $\mathrm{H}$ burning, and thus He burning via triple- $\alpha$ reactions will govern the total reaction rate, since the triple- $\alpha$ reaction rate will be the slowest rate in the nuclear flow at these high $l_{\text {acc }}$. The notion that the triple- $\alpha$ reaction rate is the slowest rate in the nuclear flow is precisely what one-zone type I X-ray burst ignition models assume (Fuiimoto et al. 1981; Paczvński 1983; Bildsten 1998; Cumming \& Bildsten 2000; Heger et al. 2005), and we suggest that this is the reason why the results of one-zone and time-dependent multi-zone models agree so well. All of these models predict that nuclear burning is thermally stable for temperatures $T \gtrsim 5 \times 10^{8} \mathrm{~K}$, which are reached only at accretion rates $l_{\text {acc }} \gtrsim 1$ (e.g., Schatz et al. 1999). Figure 11illustrates that, for relatively large values of $f_{\mathrm{rp}}$, there is a regime of prompt bursts at accretion rates greater than the accretion rates at which delayed mixed bursts occur, and the critical accretion rate above which these prompt mixed bursts cease is roughly $\dot{M}_{\text {Edd }}$, in very good agreement with these other models. Figure 1 illustrates also that nuclear burning is stable for the small range of $l_{\text {acc }}$ between these two regimes. These accretion rates are high enough to suppress delayed mixed bursts, but they are not high enough to cause a sufficient leakage out of hot CNO cycle $\mathrm{H}$ burning and trigger a thermal instability.

The model we present in this work suggests that, if the true ${ }^{15} \mathrm{O}(\alpha, \gamma){ }^{19} \mathrm{Ne}$ cross section is greater than approximately 0.1 of the CF88 rate, type I X-ray bursts should occur in systems with accretion rates near the Eddington limit. Observations indicate that this is not the case, since low-mass X-ray binaries with $l_{\text {acc }} \gtrsim 0.3$ generally do not exhibit bursts. This suggests that the true ${ }^{15} \mathrm{O}(\alpha, \gamma){ }^{19} \mathrm{Ne}$ reaction rate is less than that proposed by CF88. This conclusion is complementary to that of Fisker et al. (2006), who were the first to propose that the occurrence of type I X-ray bursts is sensitive to the strength of the ${ }^{15} \mathrm{O}(\alpha, \gamma){ }^{19} \mathrm{Ne}$ reaction rate, and who found that the existence of bursts in systems with $l_{\text {acc }} \approx 0.1$ suggests a lower bound on this rate. This lower bound corresponds to $f_{\text {rp }} \approx 0.05$ in our notation. We also note that a relatively low ${ }^{15} \mathrm{O}(\alpha, \gamma){ }^{19} \mathrm{Ne}$ reaction rate is consistent with the existence of carbon-triggered superbursts, since a low rate would increase the carbon yield resulting from stable nuclear burning (Schatz et al. 1999; Cooper et al. 2006; Fisker et al. 2006). The systems GX 17+2 and Cyg X-2 are exceptions to this empirical rule, however, for they exhibit bursts at accretion rates near the Eddington limit (e.g., Kahn \& Grindlay 1984; Tawara et al. 1984; Kuulkers et al. 2002). However, it is possible that they show bursts for other reasons, e.g., they harbor mass donor stars with H-deficient envelopes (Cooper et al. 2006).

Although the resonant contribution of the $4.03 \mathrm{MeV}$ state dominates the ${ }^{15} \mathrm{O}(\alpha, \gamma){ }^{19} \mathrm{Ne}$ reaction rate for the temperatures 
$T \lesssim 5 \times 10^{8} \mathrm{~K}$ at which helium burning can possibly trigger a type I X-ray burst, other resonances such as the 4.38 $\mathrm{MeV}$ state contribute as well. However, these resonances contribute much less than a tenth of the $4.03 \mathrm{MeV}$ contribution (e.g., Davids et al. 2003), whereas a reduction of the total ${ }^{15} \mathrm{O}(\alpha, \gamma){ }^{19} \mathrm{Ne}$ reaction rate by a factor of only $\approx 10$ is needed to stabilize nuclear burning. Therefore, it is the $4.03 \mathrm{MeV} \alpha$ width alone that determines the stability of nuclear burning at high accretion rates.

\section{CONCLUSIONS}

Using the global linear stability analysis of Cooper \& Naravan (2005), which is an expanded and improved version of the model of $\mathrm{NH} 03$, we have investigated the effects of the hot $\mathrm{CNO}$ cycle breakout reaction ${ }^{15} \mathrm{O}(\alpha, \gamma){ }^{19} \mathrm{Ne}$ on the occurrence of type I X-ray bursts on accreting neutron stars at high accretion rates. For low values of $f_{\mathrm{rp}} \lesssim 0.1$, where $f_{\mathrm{rp}}$ is a dimensionless factor by which we multiply the ${ }^{15} \mathrm{O}(\alpha, \gamma){ }^{19} \mathrm{Ne}$ reaction rate of $\mathrm{CF} 88$, the hot $\mathrm{CNO}$ cycle breakout reaction slightly lowers the critical accretion rate above which delayed mixed bursts occur, but otherwise the breakout reaction has little effect on the burst onset. The predictions of these models are in good agreement with observations. For $f_{\mathrm{rp}} \gtrsim 0.1$, a new regime of prompt mixed bursts appears at accretion rates above the rates at which delayed mixed bursts occur, and the bursts survive up to roughly the Eddington limit. The existence of this prompt mixed burst regime up to the Eddington limit is consistent with nearly all previous theoretical models. Our results support the hypothesis of $\mathrm{CN} 06$ that the discrepancies between the results of the global linear stability analysis of $\mathrm{NH} 03$ and the results of time-dependent multi-zone models with large reaction networks may be caused by the latter models assuming too large a strength for the ${ }^{15} \mathrm{O}(\alpha, \gamma){ }^{19} \mathrm{Ne}$ reaction rate at temperatures $T \lesssim 6 \times 10^{8} \mathrm{~K}$. The fact that observations agree much better with the results of $\mathrm{NH} 03$ and CN06 implies that the true reaction rate is lower than the rate assumed in these multi-zone models. Specifically, we suggest that the true $\alpha$ width $\Gamma_{\alpha}$ of the $4.03 \mathrm{MeV}$ state in ${ }^{19} \mathrm{Ne}$ is lower than the $\alpha$ width proposed by Langanke et al. (1986). Calculations using multi-zone models such as those of Wooslev et al. (2004) and Fisker et al. (2006), but with the ${ }^{15} \mathrm{O}(\alpha, \gamma){ }^{19} \mathrm{Ne}$ reaction rate lowered in strength, need to be carried out to either confirm or refute this suggestion.

We thank the referee for several insightful comments and suggestions. This work was supported by NASA grant NNG04GL38G.

\section{REFERENCES}

Ayasli, S. \& Joss, P. C. 1982, ApJ, 256, 637

Bildsten, L. 1998, in NATO ASIC Proc. 515: The Many Faces of Neutron Stars., 419

Caughlan, G. R. \& Fowler, W. A. 1988, Atomic Data and Nuclear Data Tables, 40, 283 (CF88)

Cooper, R. L., Mukhopadhyay, B., Steeghs, D., \& Narayan, R. 2006, ApJ, 642,443

Cooper, R. L. \& Narayan, R. 2005, ApJ, 629, 422

—. 2006, accepted by ApJ (astro-ph/0605001) (CN06)

Cornelisse, R., in't Zand, J. J. M., Verbunt, F., Kuulkers, E., Heise, J., den Hartog, P. R., Cocchi, M., Natalucci, L., Bazzano, A., \& Ubertini, P. 2003, A\&A, 405, 1033

Cumming, A. 2004, Nuclear Physics B Proceedings Supplements, 132, 435

Cumming, A. \& Bildsten, L. 2000, ApJ, 544, 453

Davids, B., et al. 2003, Phys. Rev. C, 67, 065808

Dewitt, H. E., Graboske, H. C., \& Cooper, M. S. 1973, ApJ, 181, 439

Fisker, J. L., Brown, E. F., Liebendörfer, M., Thielemann, F.-K., \& Wiescher, M. 2005, Nuclear Physics A, 752, 604

Fisker, J. L., Gorres, J., Wiescher, M., \& Davids, B. 2006, accepted by ApJ astro-ph/0410561,

Fisker, J. L., Hix, W. R., Liebendörfer, M., \& Thielemann, F.-K. 2003, Nuclear Physics A, 718, 614

Fujimoto, M. Y., Hanawa, T., \& Miyaji, S. 1981, ApJ, 247, 267

Fushiki, I. \& Lamb, D. Q. 1987, ApJ, 323, L55

Görres, J., Wiescher, M., \& Thielemann, F.-K. 1995, Phys. Rev. C, 51, 392

Hahn, K. I., et al. 1996, Phys. Rev. C, 54, 1999

Hansen, C. J. \& van Horn, H. M. 1975, ApJ, 195, 735

Heger, A., Cumming, A., \& Woosley, S. E. 2005, ApJ, submitted astro-ph/0511292

Hoyle, F. \& Fowler, W. A. 1965, in Quasi-Stellar Sources and Gravitational Collapse, 17

Kahn, S. M. \& Grindlay, J. E. 1984, ApJ, 281, 826

Kanungo, R., et al. 2006, Phys. Rev. C, submitted (nucl-ex/0605033
Kuulkers, E., Homan, J., van der Klis, M., Lewin, W. H. G., \& Méndez, M 2002, A\&A, 382, 947

Langanke, K., Wiescher, M., Fowler, W. A., \& Gorres, J. 1986, ApJ, 301, 629

Mitler, H. E. 1977, ApJ, 212, 513

Narayan, R. \& Heyl, J. S. 2003, ApJ, 599, 419 (NH03)

Paczyński, B. 1983, ApJ, 264, 282

Remillard, R. A., Lin, D., Cooper, R. L., \& Narayan, R. 2006, ApJ, 646, 407 Salpeter, E. E. \& van Horn, H. M. 1969, ApJ, 155, 183

Schatz, H., Bildsten, L., Cumming, A., \& Wiescher, M. 1999, ApJ, 524, 1014

Schwarzschild, M. \& Härm, R. 1965, ApJ, 142, 855

Strohmayer, T. \& Bildsten, L. 2006, in Compact Stellar X-Ray Sources, ed.

W. H. G. Lewin and M. van der Klis (Cambridge: Cambridge Univ. Press)

Taam, R. E. 1985, Annual Review of Nuclear and Particle Science, 35, 1

Taam, R. E., Woosley, S. E., \& Lamb, D. Q. 1996, ApJ, 459, 271

Tan, W. P., Görres, J., Daly, J., Couder, M., Couture, A., Lee, H. Y., Stech, E., Strandberg, E., Ugalde, C., \& Wiescher, M. 2005, Phys. Rev. C, 72, 041302

Tawara, Y., Hirano, T., Kii, T., Matsuoka, M., \& Murakami, T. 1984, PASJ, 36, 861

van Paradijs, J., Cominsky, L., Lewin, W. H. G., \& Joss, P. C. 1979, Nature, 280,375

van Paradijs, J., Penninx, W., \& Lewin, W. H. G. 1988, MNRAS, 233, 437

Wagoner, R. V. 1969, ApJS, 18, 247

Wallace, R. K. \& Woosley, S. E. 1981, ApJS, 45, 389

Wiescher, M., Görres, J., \& Schatz, H. 1999, Journal of Physics G Nuclear Physics, 25, 133

Wiescher, M., Schatz, H., \& Champagne, A. E. 1998, Royal Society of London Philosophical Transactions Series A, 356, 1

Woosley, S. E., Heger, A., Cumming, A., Hoffman, R. D., Pruet, J., Rauscher, T., Fisker, J. L., Schatz, H., Brown, B. A., \& Wiescher, M. 2004, ApJS, 151,75 